(a)

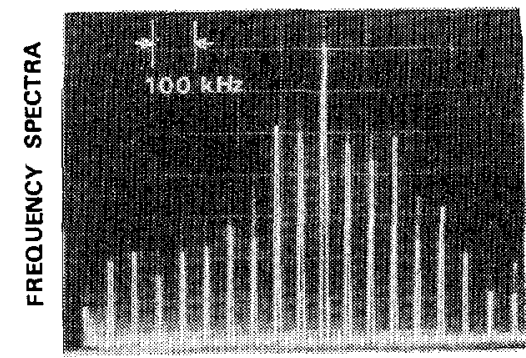

(b)

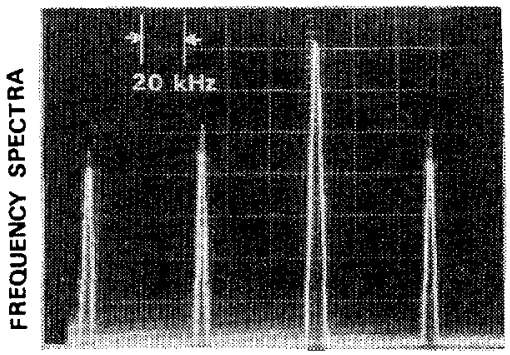

(c)

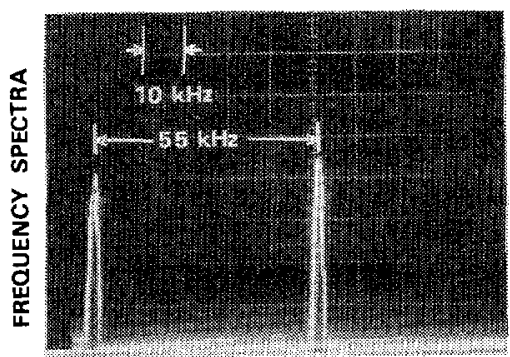

Fig. 3. The fine structure of the self-sustained oscillation spectrum. The frequency spectrum shown in trace (a) is further resolved in traces (b) and (c). fier and a step-index multimode fiber, was investigated. As a result, it is found that the spectral profile of the self-sustained oscillation follows the baseband frequency response of the fiber. If the nonlinear phenomenon in the oscillator is vanishingly small over wide oscillation frequency range, we may obtain the frequency characteristics of the wideband fiber such as graded-index multimode fibers. In addition, applying a mode-locking technique for the self-sustained oscillator, we could measure the frequency response of the system more precisely.

\section{ACKNOWLEDGMENT}

The author would like to express thanks to N. Uchida, M. Tokuda, T. Tanifuji, and Y. Koyamada for their fruitful discussions. Thanks are also due to H. Fukutomi and Y. Kato for their successive encouragement.

\section{REFERENCES}

[1] M. Nakazawa, M. Tokuda, and N. Uchida, "Self-sustained in tensity oscillation of a laser diode introduced by a delayed electrical feedback using an optical fiber and an electrical amplifier," Appl. Phys. Lett., vol. 39, pp. 379-381, 1981.

[2] W. J. Cunningham, "Nonlinear oscillators with constant time delay," J. Franklin Inst., vol. 261, pp. 495-507, 1956.

[3] V. Met, "On multimode oscillators with constant time delay," Proc. IRE, vol. 45, pp. 1119-1128, 1957.

[4] M. I. Disman and W. A. Edson, "Simultaneous asynchronous oscillations in class-C oscillators," Proc. IRE, vol. 46, pp. 895-903, 1958.

[5] I. Kobayashi and M. Koyama, "Measurement of optical fiber transfer function based upon the swept frequency technique for baseband signal," IECE Japan, vol. E59, pp. 11-12, 1976.

\title{
Zn II and As II CW Laser Transitions Excited by an Electron Beam
}

\author{
J. J. ROCCA, J. D. MEYER, AND G. J. COLLINS
}

\begin{abstract}
CW laser action was obtained on the 4911.6, 4924.0, 6102.5 , and $7588.5 \AA$ transitions of $\mathrm{Zn} \mathrm{II} \mathrm{and} \mathrm{on} \mathrm{the} 6511.7 \AA$ transition of As II using electron beam excitation of He- $\mathrm{Zn}$ and He-As mixtures, respectively.
\end{abstract}

\section{INTRODUCTION}

$\mathrm{R}^{\mathrm{R}}$ ECENTLY, we demonstrated the use of dc electron beams to excite $\mathrm{CW}$ ion lasers, obtaining $\mathrm{CW}$ laser action in singly ionized mercury [1], iodine [2], and selenium [3]. Electron beam created plasmas provide a non-Maxwellian electron energy distribution, with a high density of energetic electrons, which makes them an attractive medium for ion

Manuscript received March 30, 1982. This work was supported by the National Science Foundation.

The authors are with the Department of Electrical Engineering, Colorado State University, Fort Collins, CO 80523. lasers. We now report CW laser oscillation in singly ionized zinc and arsenic using this same electron beam pumping scheme to excite $\mathrm{He}-\mathrm{Zn}$ and $\mathrm{He}-\mathrm{As}$ mixtures. $\mathrm{CW}$ laser radiation was achieved on the $4911.6,4924.0,6102.5$, and $7588.5 \AA$ transitions of $\mathrm{Zn} \mathrm{II}$ and on the $6511.7 \AA$ transition of As II.

The laser setup used was similar to that employed previously [1] -[3]. The electron beam is injected into a plasma tube 1.1 $\mathrm{cm}$ in diameter and $100 \mathrm{~cm}$ long. An electromagnet surrounds the plasma tube, providing an axial magnetic field up to $4.2 \mathrm{kG}$ that helps to direct and confine the electron beam. The electron beam is produced by a glow discharge electron gun, and has an energy between 1 and $5 \mathrm{keV}$. We operate this glow discharge electron gun in helium at pressures up to 3 torr without differential pumping. The beam electrons are produced at the cathode by secondary emission following bombardment of the 


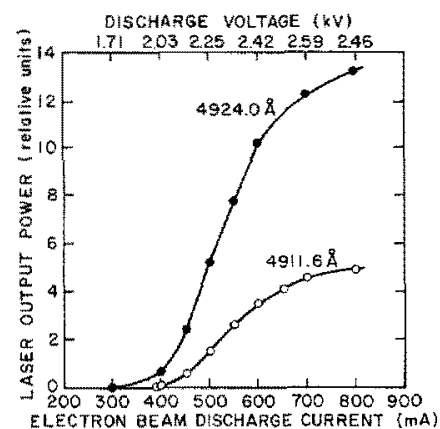

(a)

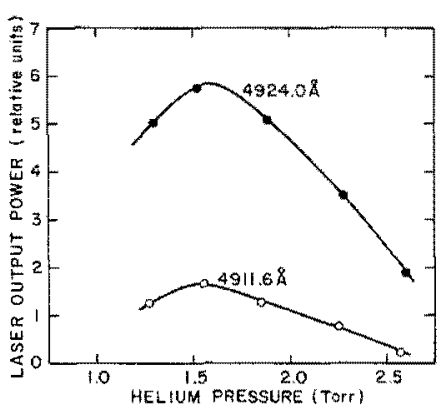

(b)

Fig. 1. (a) Laser output power of the $\mathrm{Zn}$ II blue lines as a function of electron beam discharge current and voltage. Average helium pressure in the active medium was 1.5 torr. Magnetic field was $4.2 \mathrm{kG}$. $\mathrm{Zn}$ reservoir temperature was $420^{\circ} \mathrm{C}$. (b) Laser output power of the $\mathrm{Zn}$ II blue laser lines as a function of the average He pressure in the plasma tube. Electron beam discharge current was $700 \mathrm{~mA}$ and the $\mathrm{Zn}$ reservoir temperature was $420^{\circ} \mathrm{C}$. Magnetic field of $4.2 \mathrm{kG}$.

aluminum cathode surface by ions and fast neutrals. The emitted electrons are then accelerated through the cathode dark space where practically all the voltage drop of the discharge occurs. The resulting electron beam is well collimated. Using a calorimeter we have measured an electron beam generation efficiency of 60 percent when the glow discharge is operated in helium at a pressure of 1 torr. The glow discharge electron gun provides an optical path throughout the axis [3]. This permits one to easily match the electron beam created plasma volume with the corresponding volume of an optical resonator. A metal vapor trap separates the plasma tube from the electron beam generation chamber. Metal vapor is produced by heating a source reservoir attached to the opposite end of the plasma tube. Helium is flown throughout the plasma tube at $200-400 \mathrm{~s} \cdot \mathrm{cm}^{3} / \mathrm{min}$.

The optimum reservoir temperature for $\mathrm{CW}$ laser action in zinc was found to be $420^{\circ} \mathrm{C}$. Fig. 1(a) shows the output power of the $\mathrm{Zn}$ II blue lines as a function of the electron beam discharge current and voltage at a plasma tube average helium pressure of 1.5 torr. At the largest electron beam currents the laser output power of these $\mathrm{Zn}$ II lines is observed to increase in a sublinear manner. This behavior is judged to be due to the sublinear increase of the discharge power with current, since the discharge voltage drop at $800 \mathrm{~mA}$ was measured to be smaller than at $700 \mathrm{~mA}$, as shown in Fig. 1(a). Fig. 1(b) shows the pressure dependence of the laser output power of the blue $\mathrm{Zn}$ II laser lines. The most intense laser lines in $\mathrm{Zn}$ II were the blue lines. Using an unoptimized output coupler with a reflectivity of 94.5 percent at $4900 \AA$ we measured a maximum $\mathrm{CW}$ laser output power of $60 \mathrm{~mW}$ for the combination of the 4911.6 and $4924.0 \AA$ lines. This is comparable to the highest true $\mathrm{CW}$ laser output power obtained from hollow cathode devices [5]. Optimizing the optical resonator and the design of the metal vapor trap that presently stops part of the electron beam from reaching the plasma tube an output power of a few hundred $\mathrm{mW}$ should be expected in the blue lines of $\mathrm{Zn}$ II.

Fig. 2(a) and (b) shows the laser output power dependence of the 6102.5 and $7588.5 \mathrm{~A}$ lines of $\mathrm{Zn}$ II with the electron beam current and helium pressure, respectively, at the optimum magnetic field for each line. The $7588.5 \AA$ laser line pre-

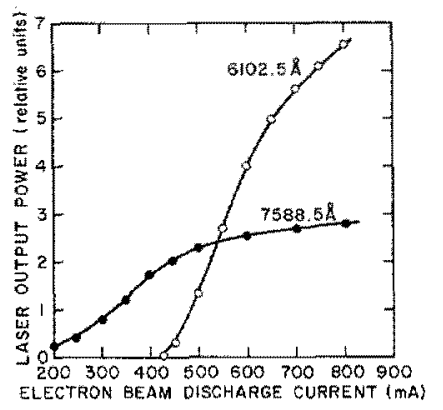

(a)

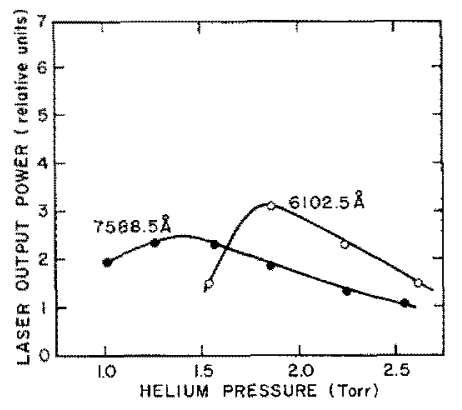

(b)
Fig. 2. (a) Laser output power of the 6102.5 and $7588.5 \AA$ lines as a function of electron beam discharge current at the optimum $\mathrm{He}$ average pressures of 1.8 torr and 1.5 torr, respectively. Magnetic field was 4.2 and $3.9 \mathrm{kG}$, respectively. $\mathrm{Zn}$ reservoir temperature was $420^{\circ} \mathrm{C}$. (b) Laser output power of the 6102.5 and $7588.5 \mathrm{~A}$ lines of $\mathrm{Zn}$ II as a function of He average pressure in the plasma tube at the optimum magnetic fields of 4.2 and $3.9 \mathrm{kG}$, respectively. $\mathrm{Zn}$ reservoir temperature was $420^{\circ} \mathrm{C}$. Electron beam discharge current was $700 \mathrm{~mA}$.

sented the lowest current threshold observed $(80 \mathrm{~mA})$, corresponding to an electron beam discharge voltage of $1.4 \mathrm{kV}$. Output power was not optimized or measured.

Fig. 3 shows the CW laser output power dependence of the $6511.7 \AA$ line of As II as a function of electron beam discharge current. The optimum arsenic source reservoir temperature was $385^{\circ} \mathrm{C}$ and the optimum helium pressure in the plasma tube was 1.3 torr. To obtain $\mathrm{CW}$ laser action on arsenic we used an optical resonator composed by two $2 \mathrm{~m}$ radius of curvature total reflectors. The laser output power was considerably smaller than that in the Zn II study and no attempt was made to optimize it.

The dominant excitation mechanism in both lasers is a thermal charge transfer reaction [7] between helium ground state ions and the metal vapor atoms. This results in formation of excited metal vapor ion levels from which the laser action originates. Note that $5 p^{2} P_{3 / 2}^{0}$ upper level of the $7588.5 \AA$ line of $\mathrm{Zn} \mathrm{II}$ is mainly populated by radiative cascade from the higher $\mathrm{Zn}$ II energy levels populated by the charge transfer process [7]. Supporting these above arguments we found that no laser action was obtained when we replaced $\mathrm{He}$ by $\mathrm{Ne}$ in the $\mathrm{He}-\mathrm{Zn}$ mixture. 


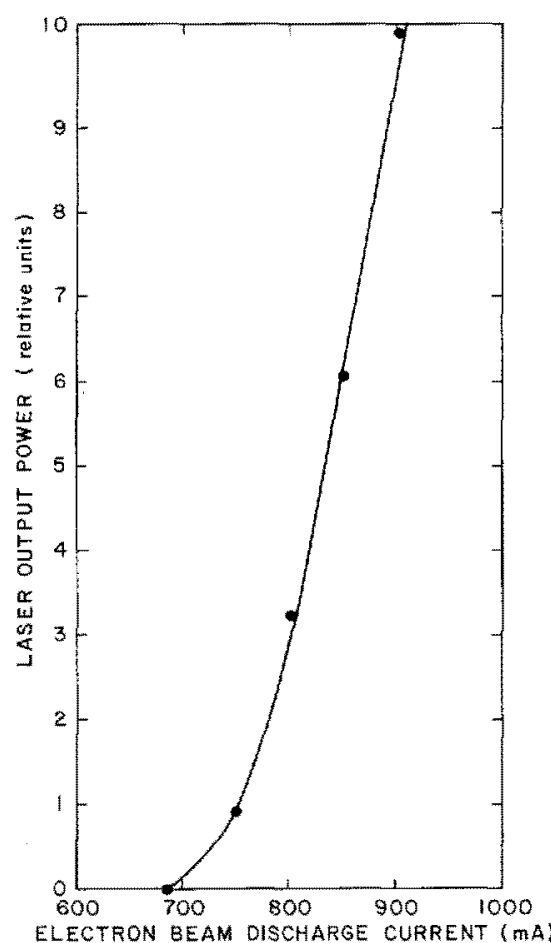

Fig. 3. Variation of the laser output power of the $6511.7 \AA$ line of As II as a function of electron beam discharge current. The As reservoir temperature was $387^{\circ} \mathrm{C}$ and the magnetic field was $4 \mathrm{kG}$. The helium average pressure in the plasma tube was 1.3 torr. The electron beam energy at $900 \mathrm{~mA}$ was $2.7 \mathrm{keV}$.

\section{CONCLUSION}

In summary, we have obtained CW laser action on $\mathrm{Zn} \mathrm{II} \mathrm{and}$ As II using for the first time electron beam excitation. We have demonstrated, as recently suggested [6], that dc electron beam pumping can be used to excite a large variety of ion lasers.

\section{ACKNOWLEDGMENT}

The authors wish to thank T. Burnell for skilled technical assistance.

\section{REFERENCES}

[1] J. J. Rocca, J. D. Meyer, and G. J. Collins, "Electron beam pumped CW Hg ion laser," Appl. Phys. Lett, vol. 40, pp. 300-302, Feb. 1982 .

[2] J. D. Meyer, J. J. Rocca, Z. Yu, and G. J. Collins, "CW iodine laser excited by an electron beam," IEEE $\mathcal{J}$. Quantum Electron, vol. QE-18, pp. 326-327, Mar. 1982.

[3] J.J. Rocca, J. D. Meyer, and G. J. Collins, "Electron beam pumped CW Se II laser," Opt. Commun, to be published.

[4] J. J. Rocca, J. D. Meyer, and G. J. Collins, "Hollow cathode electron gun for the excitation of CW lasers," Phys. Lett., vol. 87A, pp. 237-239, Jan. 1982 .

[5] J. A. Piper and P. Gill, "Output characteristics of the He- $\mathrm{Zn}$ laser," J. Phys. D. (Appl. Phys.), vol. 8, pp. 127-134, Feb. 1975.

[6] J. J. Rocca, J. D. Meyer, Z. Yu, and G. J. Collins, "Electron beam excitation of CW ion lasers," presented at the 34th Gaseous Electronic Conf., Boston, MA, Oct. 1981.

[7] G. J. Collins, "Excitation mechanism in He-Cd and He- Zn ion lasers," J. Appl. Phys, vol. 44, pp. 4633-4652, Oct. 1973.

\title{
Collisional Narrowing in the Optically Pumped $\mathrm{CH}_{3} \mathrm{OH}$ and $\mathrm{CH}_{3} \mathrm{~F}$ Lasers
}

\author{
NABIL M. LAWANDY AND GERHARD A. KOEPF
}

\begin{abstract}
The gain linewidth of the optically pumped $\mathrm{CH}_{3} \mathrm{~F}$ laser is observed to narrow and rebroaden with the addition of He. In addition,

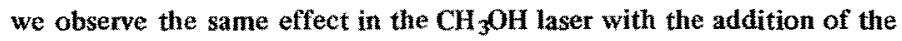
polyatomic buffer gases $\mathrm{SF}_{6}$ and $\mathrm{CS}_{2}$. These results offer conclusive evidence of the Dicke narrowing phenomena in these inverted pure rotational transitions. The effect is observed using a high harmonic mixing technique in a Schottky barrier diode.
\end{abstract}

\section{INTRODUCTION}

$I_{n}^{N}$ N 1953, Dicke discussed the effect of collisions which did not disturb the internal emission process of a radiator on the Doppler width of the transition observed [1]. He showed that in the limit that the radiator suffered many velocitychanging collisions during the time it took to travel a distance $\lambda / 2 \pi$ ( $\lambda$ is the wavelength of the emission), the Doppler width

Manuscript received February 12, 1982. This work was performed at the Goddard Space Flight Center and supported by the National Aeronautics and Space Administration.

N. M. Lawandy is with the Division of Engineering, Brown University, Providence, RI 02912.

G. A. Koepf is with COMSAT Laboratories, Clarksburg, MD 20841. would be greatly reduced. The connection between the Doppler width and the mean velocity is made through the uncertainty principle. The Doppler shift of a photon can only give information related to displacements greater than $\lambda / 2 \pi$. Therefore, when collisions occur with a mean free path less than $\lambda / 2 \pi$, the resulting Doppler information does not represent the thermal velocity, but approaches the mean of zero for zeromean free path.

The collisional narrowing effect has been experimentally verified since 1956 when Wittke and Dicke performed experiments on the $1420.4 \mathrm{MHz}$ hyperfine splitting of atomic hy. drogen [2]. In their experiment, they were able to observe the linewidth collapse by a factor of ten under optimum conditions. Subsequent to this work, other microwave measurements were carried out [3]-[7]. The experiments which followed these were at higher infrared and invisible wavelengths. The optical techniques were of a light-scattering nature, while the infrared measurements were performed using absorption spectroscopy [8], [9].

The experiments discussed so far all utilized inert gases as collision partners. In fact, attempts to observe the effect using 